\title{
Theoretical Modeling of Tunable Three-Section Slotted Fabry-Perot Lasers
}

\author{
Jialin Zhao ${ }^{1}, \mathrm{Kai}_{\mathrm{Shi}}{ }^{2}$, Yonglin $\mathrm{Yu}^{1 *}$, Member, IEEE, Liam P. Barry ${ }^{2}$, Senior Member, IEEE \\ ${ }^{1}$ Wuhan National Laboratory for Optoelectronics, School of Optical and Electronic Information, Huazhong University of Science \\ and Technology, Wuhan 430074, P. R. China, \\ ${ }^{2}$ The Rince Institute, Dublin City University, Dublin 9, Ireland. \\ * Email: yonglinyu@mail.hust.edu.cn
}

\begin{abstract}
We present a time-domain traveling wave dynamic model for simulation of wavelength tunable three-section slotted Fabry-Perot semiconductor lasers. The slot structure is characterized by using the boundary conditions of the optical fields propagating through the slot facets. Static and dynamic wavelength switching characteristics are simulated. Good agreements have been found between the simulation results and the experimental measurements.
\end{abstract}

Keywords-Slotted Fabry-Perot Laser, modeling, time-domain, wavelength switching.

\section{INTRODUCTION}

Wavelength tunable semiconductor lasers are attractive in various application, such as dense wavelength division multiplexed (DWDM) systems, optical transmission networks [1]. In the past few years, new types of laser structures fabricated by etching discrete perturbing slots into the FabryPerot laser ridge were proposed and demonstrated [2-5]. The merits of the slot structure are its fabrication simplicity and low cost as there is no epitaxial re-growth in the fabrication process and only standard photolithography is required for the approximately $1 \mu \mathrm{m}$ wide slots. Among the different kinds of SFP lasers, the three section SFP (3s-SFP) laser is attractive as only two single slots were etched into the laser ridge. And discrete tuning over 25 available channels of the $100 \mathrm{GHz}$ ITU grid were achieved with the SMSR greater than $30 \mathrm{~dB}$ and the linewidth less than $800 \mathrm{kHz}[4,5]$.

As shown in Fig. 1, the 3s-SFP laser consists of three active sections separated by two single slots etched into the laser ridge. The width of the slots is $1 \mu \mathrm{m}$. Both facets of the device are cleaved to form three FP cavities. By changing the current injected into each section, the lasing wavelength can be tuned.

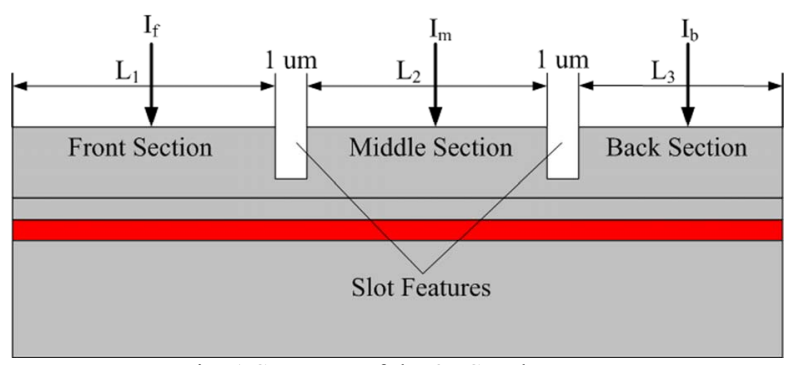

Fig. 1 Structure of the 3s-SFP laser.
Recently, we developed a time-domain traveling-wave (TDTW) model to simulate the $3 \mathrm{~s}-\mathrm{SFP}$ laser. Both the static and dynamic characteristics of this laser are simulated [6] .

\section{SiMUlation Model}

The TDTW model is based on solving the time-dependent coupled-wave equations at discrete points along the laser cavity [7]. The cavity are divide into subsections with length of $\Delta \mathrm{s}$. The forward and backward traveling optical fields in the waveguide are represented by $F$ and $R$ respectively. Fig. 2 depicts the discretization scheme of this model and the reflection and transmission of the optical field at both facets of a slot, where $r_{f}^{ \pm}$and $r_{b}^{ \pm}$represent the reflectivity of the front and back facet, respectively and the superscripts "-."and "+" represent reflectivity looking from the left and right side of a facet, respectively. $n$ and $m$ denote the time and space step, respectively. The transmissivities of the facets are represented as $t_{f}$ and $t_{b}$. The boundary conditions of the optical fields at the left and right facets can be described as:

$$
\left\{\begin{array}{l}
F_{m}^{n}=t_{f} F_{m}^{-n}+\left(-r_{f}^{+}\right) R_{m}^{+n} \\
R_{m}^{n}=r_{f}^{-} F_{m}^{-n}+t_{f} R_{m}^{+n} \\
F_{m+1}^{n}=r_{b}^{+} R_{m+1}^{+n}+t_{b} F_{m+1}^{-n} \\
R_{m+1}^{n}=t_{b} R_{m+1}^{+n}+\left(-r_{b}^{-}\right) F_{m+1}^{-n}
\end{array}\right.
$$

where the superscripts "-" and "+" in the optical field represent optical field in the left and right side of a slot facet, respectively.

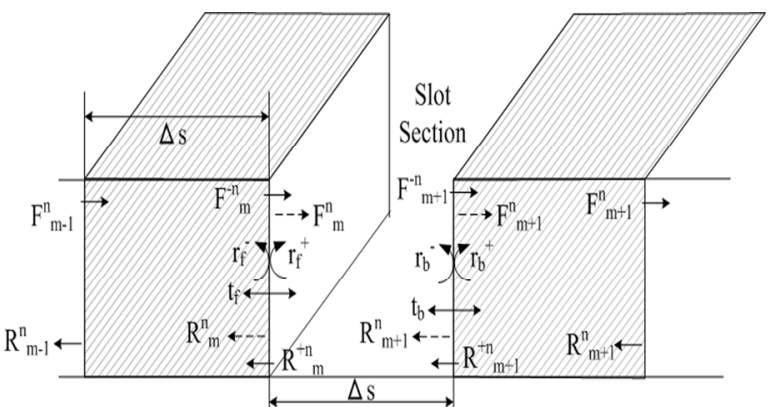

Fig. 2 Discretization scheme for the TDTW model and the reflections and transmissions at both facets of a slot. 


\section{RESULTS AND ANALYSIS}

The parameters of the 3s-SFP laser structure used in the simulation are obtained from [3, 4]. Fig. 3(a) and Fig. 3(b) show the wavelength and the SMSR tuning curves as a function of the current applied to the front section and the total current applied to both the middle and the back section equally, respectively. It is clear that the good SMSR ( $>40 \mathrm{~dB})$ can be achieved in the center area of each mode and the wavelength tuning range is found to be approximately $12 \mathrm{~nm}$ from $1543 \mathrm{~nm}$ to $1555 \mathrm{~nm}$. Fig .4 plots the superimposed spectra of the eight lasing modes from $\lambda_{2}$ to $\lambda_{9}$ in Fig. 3(a). As shown in Fig. 4, the output mode power increases with the increase of the tuning current. The mode power variation is found to be approximately $6 \mathrm{~dB}$.

The wavelength switching dynamics are simulated and shown in Fig. 5. By changing the current applied on the front section, the emitted wavelength is switched between mode $\lambda_{2}$ to mode $\lambda_{8}$ [see Fig. 3(a)] and the switching time is approximately $2.6 \mathrm{~ns}$ for forward switching and 4.6ns for backward switching. These results are in qualitative agreement with experimental results presented in [4].
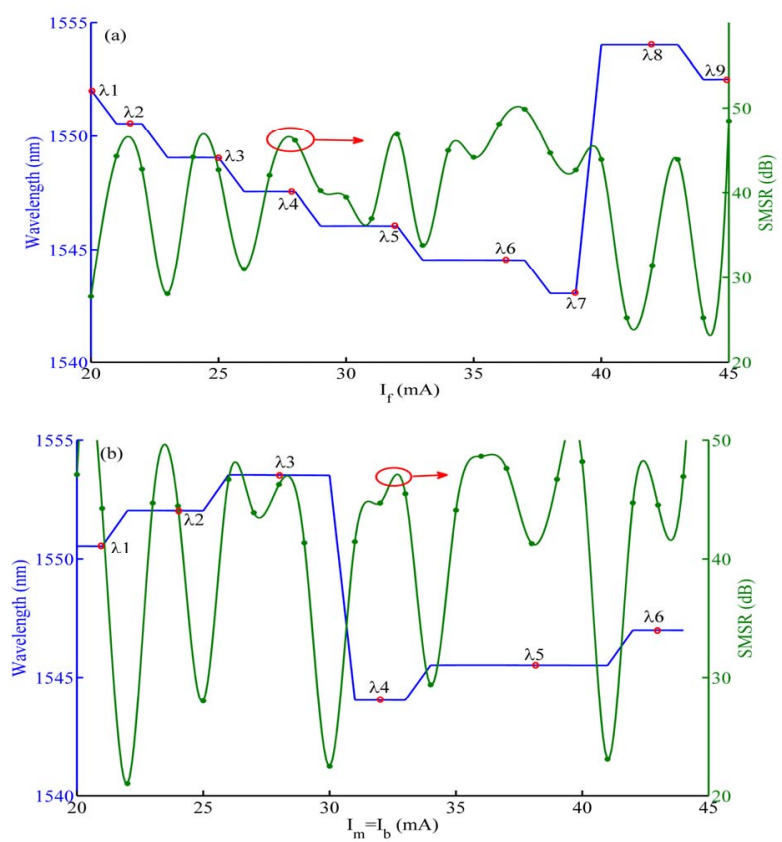

Fig. 3 Wavelength (blue line) and SMSR (green line) tuning curves versus (a) current across the front section and (b) total current across both the middle and the back sections equally.

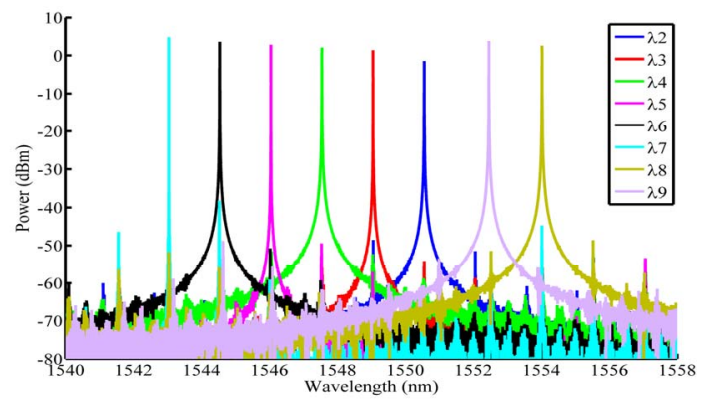

Fig. 4 Superimposed spectra of eight modes

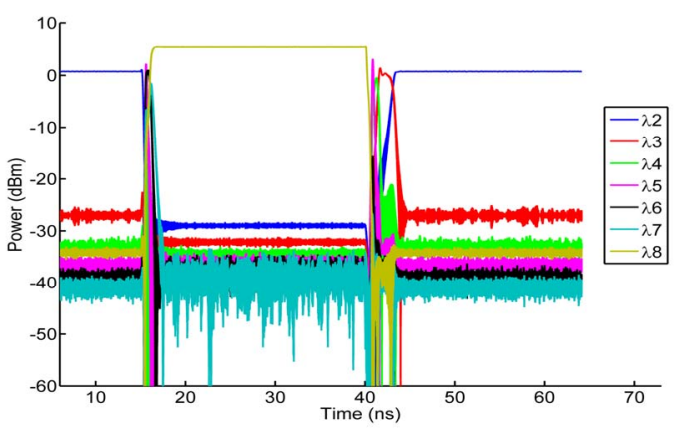

Fig. 5 Switching transients of the SFP laser between $\lambda_{2}$ to $\lambda_{8}$.

\section{CONCLUSIONS}

In this paper, we have developed a comprehensive timedomain traveling-wave model for the simulation of the $3 \mathrm{~s}-\mathrm{SFP}$ laser. Both static and dynamic performances have been investigated. Simulated results have been found to be in good agreement with the experimental measurements, which show that this model is a useful and suitable tool to study the characteristics of the 3s-SFP lasers, and also to design devices with specifically required characteristics.

\section{ACKNOWLEDGMENT}

The authors acknowledge the support provided by International S\&T Cooperation Program of China (1016), the National Natural Science Foundation of China (11174097), the Specialized Research Fund for Doctoral Program of Higher Education (20100142), the Science Foundation Ireland Principal Investigator Programme, and the Enterprise Ireland Innovation Partnership Programme.

\section{REFERENCES}

[1] J. Buus and E. J. Murphy, "Tunable lasers in optical networks," IEEE J. Lightw. Technol., vol. 24, no. 1, pp. 5-11, Jan. 2006.

[2] S. Osborne, S. O’Brien, K. Buckley, R. Fehse, A. Amann, J. Patchell, B. Kelly, D. R. Jones, J. O'Gorman, and E. P. O'Reilly, "Design of singlemode and two-color Fabry-Perot lasers with patterned refractive index," IEEE J. Sel. Topics Quantum Electron., vol. 13, no. 5, pp. 1157-1163, Apr. 2007.

[3] S. O. Brien, F. Smyth, K. Shi, J. O. Carroll, P. M. Anandarajah, D. Bitauld, S. Osborne, R. Phelan, B. Kelly, J. O. Gorman, F. H. Peters, B. Roycroft, B. Corbett, and L. Barry, "Design, Characterization, and applications of index-patterned Fabry-Perot Lasers," IEEE J. Sel. Topics Quantum Electron., vol. 17, no. 6, pp. 1621-1631, Nov/Dec. 2011.

[4] K. Shi, F. Smyth, D. Reid, C. Browning, B. Roycroft, B. Corbett, F. Peters, and L. Barry, "Fast switching slotted Fabry-Perot laser for phase modulated transmission systems," IEEE J. Lightw. Technol., vol. 28, no. 23, pp. 3409-3416, Dec. 2010.

[5] K. Shi, F. Smyth, D. Reid, B. Roycroft, B. Corbett, F. H. Peters and L. P. Barry, "Characterization of a tunable three-section slotted Fabry-Perot laser for advanced modulation format optical transmission," Opt. Comm., vol. 284, no. 6, pp. 1616-1621, Mar. 2011.

[6] J. L. Zhao, K. Shi, Y. L. Yu, and L. P. Barry, "Theoretical analysis of three-section slotted Fabry-Perot Lasers based on time-domain travelingwave model," IEEE J. Sel. Topics Quantum Electron., in press.

[7] L. M. Zhang, S. F. Yu, M. C. Nowell et al, "Dynamic analysis of radiation and side-mode suppression in a second-order DFB laser using time-domain large-signal traveling wave model," IEEE J. Quantum Electron., vol. 30, no. 6, pp. 1389-1395, Jun. 1994. 